\title{
SAFETY GUIDELINES FOR THE USAGE OF LASERS IN THE FIELD OF UROLOGY
}

\author{
Kei Matsuoka, MD.
}

Department of Urology, Kurume University School of Medicine

\section{Guidelines for the use of high output lasers in the field of urology}

Clinical conditions indicating laser treatment in the field of urology are extragenital tumors, urinary tract epithelial tumors, benign prostate hypertrophies, urinary tract strictures and urinary tract stones. These conditions are treated with high output lasers such as Neodymium doped YAG (Nd:YAG) lasers, carbon dioxide (CO2)lasers, Holmium doped YAG (Ho:YAG) lasers, potassium titanyl phosphate (KTP) frequencydoubled Nd:YAG lasers, pulsed-dye lasers and Alexandrite lasers. These high output lasers differ in tissue absorption depths and absorption coefficients for water and hemoglobin according to their respective wavelengths, therefore one must be fully aware of the characteristics of the wavelength. If and when the full capability of a laser is used properly, the patient will benefit from efficient and effective treatment with lasers, but when misused the patient is placed at risk, and may even suffer severe damage.

From this standpoint, the purpose of this article is to set a series of "Safety Guidelines" for the proper use of lasers, thereby ensuring the safety of both the patient and the operator during the use of lasers in the field of urology.

\section{The requirements of the medical facility and medical doctors for the use of high output lasers.}

The medical facility is required to have medical personnel well trained in lasers belonging to the facility

\section{Addressee for Correspondence:}

Kei Matsuoka, MD.

Dep. of Urology, Kurume University School of Medicine 67 Asahimachi Kurume-shi Fukuoka-ken

JAPAN 830-0011

Tel: +81-942-35-3311

Email: keimat@med.kurume-u.ac.jp and associated laser treatment techniques. The facility should be certified by the the non-profit organization, the Japan Society of Laser Surgery and Medicine and an hold certification as an Educational Facility, certified by the Japanese Urological Association. The medical doctor operating the laser is required to be a boardcertified specialist from both of the above-mentioned societies.

\section{Indications for high output laser treatment}

\section{Extragenital tumors}

The Nd:YAG laser treatment of genital warts (condylomata acuminata) is very effective, however the high degree of tissue coagulation of this laser necessitates caution regarding too much laser energy reaching the urethra. There have been previous reports of severe complications such as exfoliation and necrosis of the urethra. ${ }^{(1)}$

Laser treatment of penile cancers seems to be a promising means to avoid radical penile resection. For superficial cancers the $\mathrm{CO} 2$ laser is used and for infiltrative cancers the combination of the Nd:YAG laser with the $\mathrm{CO} 2$ laser is preferred. There is a report that this combination was both functionally and appearance-wise better than conservative penile resection. (2)

\section{Urinary tract superficial cancers}

The Nd:YAG laser treatment of bladder cancer holds merits such as no bleeding, no anesthesia required, flexible endoscopes may be used, no possibility of bladder rupture, no stimulation of the obturator nerve, and no need to place balloon catheters. However there is no evidence concerning the lowering of the recurrence rate of the tumor compared to conventional

Manuscript received: August 2008

Accepted for publication: September 2008

Laser Therapy 17.3: 155-158 
treatments. Recently, attempts at treating superficial bladder cancers using the Ho:YAG laser which has a higher absorption coefficient in water, and thus better tissue absorption and a lower penetration depth, have been made. (3) This method is the same in context of a outpatient treatment and there is no need for indwelling catheters as in the Nd:YAG laser treatment, but differs in the laser tissue interaction whereby the Ho:YAG laser is used in vaporization and cutting of the tumor base compared with the tissue and tumor coagulation with the Nd:YAG laser. The Ho:YAG laser should be studied in comparison with conventional transurethral bladder tumor resection (TUR-Bt) using high frequency electro-cauterization, in a prospective trial. Attempts at treatment of bladder tumors with the high out-put diode lasers have also been made.

Endoscopic transurethral treatment of urethral and/or renal pelvis tumors is an efficacious treatment method if the indications are strictly defined. Conventionally the Nd:YAG laser has been used, but recently the Ho:YAG laser has been used, resulting in the safer and more efficient vaporization, cutting and coagulation of tumors due to the lower degree of scattering in tissues associated with the Ho:YAG wavelength.$(4,5)$

\section{Benign prostate hypertrophy $(\mathrm{BPH})$}

There was a time when the Nd:YAG laser was used for the treatment of $\mathrm{BPH}$ in the non-contact method through a lateral emission fiber, but the time for the coagulated tissue to be absorbed took 4-6 weeks which meant that any subjective and/or objective improvement of symptoms took just as long. However, complications were still substantially fewer than with the conventional TUR-P. (6) The contact method of laser treatment using the same lateral emission fibers differs in the laser-tissue interaction from the non-contact method in that the contact method creates a cavity within the prostate through vaporization of the tissue, in contrast to tissue coagulation associated with the non-contact method. The creation of the cavity results in immediate alleviation of difficulties in urination. The time it takes for this treatment method is longer, and should be done on prostates with volumes of less than $60 \mathrm{ml}$. Interstitial laser irradiation treatment is a treatment whereby the prostate tissue is deliberately punctured by a bare laser fiber either transurethrally or via the perineum. This procedure takes very little time, can be performed under local anesthesia in an outpatient setting and allows safe treatment for highrisk patients and patients with complications. (7) The drawback of this method is the same as the non-con- tact trans-urethral Nd:YAG laser treatment, where tissue absorption and shrinkage is time consuming and alleviation of symptoms only appears thereafter.

Recently, the Ho:YAG and the KTP frequencydoubled YAG lasers have also been used for safe and effective treatments. Holmium laser enucleation of the prostate (HoLEP) using a direct emission fiber is an effective method for treatment of large volume prostates. ${ }^{(8)}$ Holmium laser ablation of the prostate (HoLAP) using lateral emission fibers is also a safe and effective treatment method. Treatment of the prostate using the KTP frequency-doubled YAG laser for its vaporization capabilities is a novel method. Laser is emitted through a lateral emission fiber in this case. ${ }^{(9)}$

\section{Urinary tract stenosis and strictures}

For stenosis and strictures of the ureteropelvic junction, percutaneous antegrade pyelotomy used to be the main method of treatment, but since the appearance of lasers on the medical scene, transurethral retrograde endopyelotomies using the Nd:YAG and the Ho:YAG lasers have been performed. The latter laser is widely used and safe. However concerning retrograde endopyelotomy, studies report no difference in efficacy of the treatment between Ho:YAG laser incision and electrocautery incisions. ${ }^{(10)}$

For urethral stenosis, numerous lasers such as the Nd:YAG, carbon dioxide, argon and the KTP frequency-doubled YAG lasers have been used. The use of the Nd:YAG laser in the pulsed mode has been reported as having a good treatment record. (11) It is believed that the pulsed mode minimizes tissue coagulation and increases tissue vaporization compared to the continuous wave emission, leading to better results. ${ }^{(12)}$ For posterior urethral valves, small diameter urethroscopes and Nd:YAG or Ho:YAG lasers are used.

\section{Urinary tract stones}

Historically, treatments with numerous types of lasers have been attempted. Lasers which have been widely used clinically are the pulsed dye laser ${ }^{(13)}$, alexandrite laser (14) and Ho:YAG laser (15). In terms of lithotripsy, the Ho:YAG laser is superior to the others.

\section{Endoscopy during the use of high output lasers}

Laser energy is guided to the target tissues using rigid or flexible cystoscopes, rigid or flexible renoureteroscopes, renoscopes for percutaneous nephrolithotripsy, and urethrascopes for treatment of the prostate. The selection of the most appropriate endoscopes must be made according to the treatment. 


\section{Instructions for laser emission during endo- scopic procedures}

In the field of urology, laser treatment has been historically well- associated with endoscopic procedures. Therefore in most cases lasers are fired while submerged in perfusion fluid. One must take care to make sure the fluid is being perfused at all times while the laser is being emitted. Stasis of the fluid during laser irradiation may cause heating of the surrounding tissue. Also when there are air bubbles in the field of laser irradiation, do not fire lasers while the air bubbles are present. Especially during the use of the Ho:YAG laser, whose absorption coefficient in water is comparatively high, the laser tissue interaction will differ when the laser is emitted through water and when the laser is emitted through air. The latter will cause a much more intense tissue reaction.

From the standpoint of maintenance of the endoscopic equipment, the laser fibers should protrude from the tip of the endoscope during laser irradiation. Prior to laser irradiation, make sure that both the tip of the laser fiber and the target tissue are visible within the same visual field. Never fire lasers without confirming the location of the laser fiber tip.

When using an endoscope for laser irradiation, treat the silica fiber of the laser with care while inserting the fiber into the endoscope. During the procedure, be careful to leave a certain amount of slack for the silica fiber to compensate for the movement of the endoscope.

\section{Instructions for post-laser treatment care and management following treatment of renoureteral cancers, urinary tract stenosis and upper urinary tract stones.}

Following laser treatment of ureteric cancers, ureteropelvic junction stenosis, ureteric stenosis, urethral stenosis and ureteric stones, post-treatment observations either through imagery and/or endoscopy should be performed not only to follow-up the lesion but also to rule out any new stenotic lesions and recurrences.

\section{Securing the safety of the medical personnel}

Strict adherence to instructions concerning the maintenance and management of the laser hardware is required. Lasers are to be operated only when protective goggles, suited for the respective wavelength, are worn by all involved in the procedure.

\section{Informed consent}

An signed form of informed consent from the patient is required prior to the laser treatment. The patient must be fully informed regarding the indications and the actual procedure of the treatment in comparison to other treatment modalities, treatment results at that specific facility, associated risks and complications, in addition to the benefits of the treatment.

\section{Changes in the guidelines}

In order to provide an evidence based, high quality and safe treatment, these guidelines will be revised as and when required.

6: Kabalin J.N. Laser coagulation. In Smith S.D.et al ed. Smith's Textbook of Endourology. Quality Medical Publishing. St. Louis. 1996; Vol.2: 10781099

7: Rosette J.J., Muschter R., Lopez M.A. et al. Interstitial laser coagulation in the treatment of benign prostatic hyperplasia using a diode-laser system with temperature feedback. Br J Urol 1997; 80: 433-438

8: Gilling P.J., Kennett K.M., Fraundorfer M.R. Holmium laser enucleation of the prostate for glands larger than 100g: An endourologic alternative to open prostatectomy. J Endourol 2000; 14: 529-531

9: Carter A., Sells H., O’Boyle P.J. High-power KTP 
laser for the treatment of symptomatic benign prostatic enlargement. BJU Int 1999; 83: 857-858

10: Gerber G.S., Kim J.C. Ureteroscopic endopyelotomy in the treatment of patients with ureteropelvic junction obstruction. Urology 2000; 55: 198-202

11: Merkle W. Urethra In Smith JA et al (ed) Lasers in urologic surgery. Mosby, St. Louis, Chapt. 5, 1994; 44-55

12: Vincente J., Salvador J., Caffaratti J. Endoscopic urethrotomy versus urethrotomy plus Neodymium:YAG laser int the treatment of urethral stric- tures. Eur Urol 1990; 18:166-168

13: Watson G.M., Dretler S., Parrish J.A. The pulsed dye laser for fragmenting urinary calculi. J Urol 1987; 138: 195-198

14: Mattioli S., Cremona M., Benaim G. et al. Lithotripsy with a Q-switched alexandrite laser system. Preliminary in vivo and in vitro results. Eur Urol 1991; 19: 233-235

15: Matusoka K., Iida S., Nakanami M. et al. Holmium: yttrium-aluminum- garnet laser for endoscopic lithotripsy. Urology 1995; 45: 947-952 